\title{
Design of a Rectangular-Type Voice Coil Actuator for Frame Vibration Compensation
}

\author{
Young-Man Choi ${ }^{1}$, Dahoon Ahn ${ }^{2}$, Dae-Gab Gweon ${ }^{3}$, and Moon Gu Lee ${ }^{1 *}$ \\ ${ }^{1}$ Department of Mechanical Engineering, Ajou University, Republic of Korea \\ ${ }^{2}$ High-speed Railroad Systems Research Center, Korea Railroad Research Institute, Republic of Korea \\ ${ }^{3}$ Department of Mechanical Engineering, Korea Advanced Institute of Science and Technology, Republic of Korea
}

(Received 5 April 2016, Received in final form 24 August 2016, Accepted 25 August 2016)

\begin{abstract}
Precision motion stages used in the manufacturing process of flat-panel displays have inevitably low settling performance due to their huge mass and bulky structures. In order to improve the settling performance, several methods of frame vibration compensation have been developed so far. These methods are used to cancel the vibration by imposing a counter force or modifying the resonance mode of the frame of the stage. To compensate the frame vibration, high force actuators are required. In this paper, a mighty voice coil actuator is proposed to generate the counter force against the frame vibration. The proposed voice coil actuator has an axis-symmetric rectangular structure to achieve a large force with simple and low cost fabrication. Also, the voice coil actuator allows radial clearance up to $\pm 4 \mathrm{~mm}$. Using an optimized design process and a magnetic circuit model, the power consumption is minimized while the required force is obtained. With a power of 322 W, the VCA has been designed to have a maximum force of $574 \mathrm{~N}$ with a force constant of 164 N/A. Experimental results verified the force constant of the fabricated voice coil actuator is well matched with the designed value.
\end{abstract}

Keywords : active vibration isolation, voice coil actuator, precision stage

\section{Introduction}

For the last two decades, rapid evolution of flat panel display (FPD) has accompanied a continuous progression of long-stroke precision stages that are used in transportation and manufacturing of the FPD. Nowadays, some companies are focusing on the development or sale of the ultra-large stage corresponding to $11^{\text {th }}$ generation panel $(3050 \mathrm{~mm} \times 3450 \mathrm{~mm})$. The stage has long strokes spanning several meters and has a high accuracy in the order of a few micro-meters. High speed operation of the ultra-large stage cause a long settling time that it need to wait for the next manufacturing process.

The settling time is determined by two critical factors, one being the bandwidth of the servo system. If the bandwidth frequency is low, the response of the stage becomes slow, a long settling time becomes inevitable, and the capability of the stage to reject disturbances weakens. The

CThe Korean Magnetics Society. All rights reserved.

*Corresponding author: Tel: $+82-31-219-2338$

Fax: +82-31-219-1611, e-mail: moongulee@ajou.ac.kr other critical factor is the low resonance frequency of the frame. The frame is a part on which the moving mechanism with the flat panel are placed. Generally, the frame is a heavy steel or granite plate and is vibration-isolated by using passive vibration isolators. In order to sufficiently attenuate the ground vibration, the frame has a low resonant frequency typically in range of 2-10 Hz. This resonance is induced when the stage accelerates or decelerates, and the residual vibration of the frame is then transmitted to the working frame: that has working tools, i.e. inspection camera, coating head and printing nozzle. This frame vibration of the working frame slows down the process and increases the settling time.

Rankers [1] has listed several guidelines to reduce the vibration of the frame, among which the frame vibration compensation is an active and effective method. This method compensates the reaction force with additional actuators by measuring the acceleration of the stage. Canon [2] and Nikon [3] have used this method for high precision stages equipped in the lithography devices. To generate the compensating force, various servomechanism actuators such as piezoelectric actuator [4], electromagnetic 
actuator [5, 6], giant magnetostrictive (GMS) actuator [7], and air servo valve [8] have been developed. Because a piezoelectric or a GMS actuator requires a rigid connection between base and the frame, they have a higher resonant frequency that makes the stage vulnerable to ground vibrations. Air servo valves have little effect on the resonant frequency but they have slow response. An electromagnetic actuator is mechanically decoupled without a direct connection and has a high bandwidth with easy control. However, the heat generated while producing a large force can be a limitation. The power consumption of the electromagnetic actuator should not exceed the critical temperature (the melting point) of the coil coating or the working temperature of permanent magnets. Among electromagnetic actuators, a voice coil actuator that uses Lorentz force has been studied for various applications such as hard disk drive [9], vibration isolation [5, 6], lens focusing [10], high-precision stage [11] and fast-steering mirror [12]. In comparison with a solenoid actuator, the voice coil actuator has a somewhat lesser force capacity but a remarkable linearity between the current and force. Since the non-linearity of an actuator increases the control complexity and requires a nonlinear control algorithm, with most of the industrial controllers of simple proportional-integral-derivative (PID) control, it leads to a poor control performance. Moreover, the voice coil actuator can move in both directions by simply alternating the direction of current.

In this paper, we propose a voice coil actuator (VCA) for compensation of frame vibration. The proposed voice coil actuator is designed to work together with passive vibration isolation system. Because the passive vibration isolation system has very low stiffness in all directions, the frame vibrates not only in the stage-moving direction but also in the other directions. The proposed VCA was designed to have enough radial clearance. Also, it has an axis-symmetric structure that can produce a large force per unit volume. Through design optimization, the power consumption is minimized with the allowance that the generated force is large enough to overcome the reaction by the stage's acceleration. The organization of the rest of the paper is as follows: Section 2 presents the conceptual design of the proposed VCA while Section 3 discusses its design optimization. In Section 4, the force generated by the voice coil actuator is evaluated, and conclusions are reported in Section 5.

\section{Conceptual Design}

The cross-sectional view of the proposed VCA is shown in Fig. 1, depicting a symmetric structure. For the VCA to

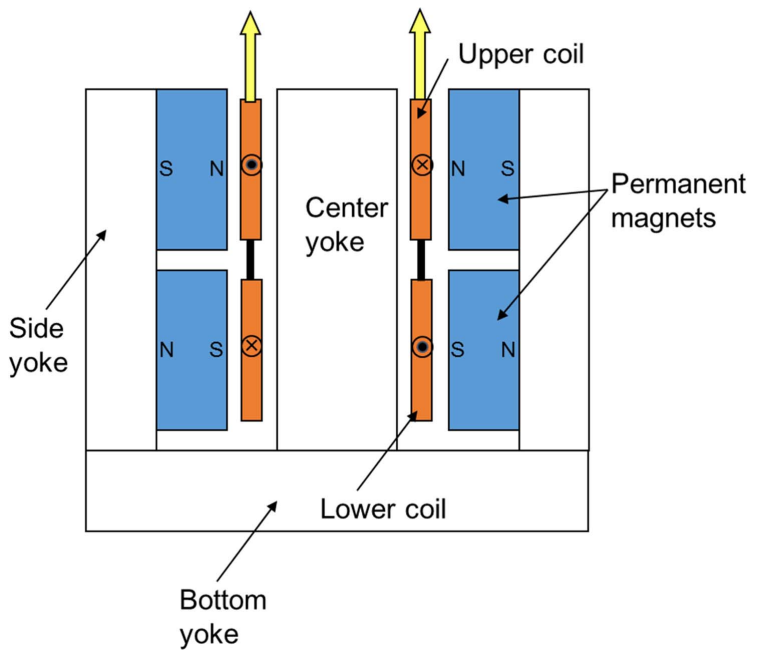

Fig. 1. (Color online) Cross-sectional view of the proposed voice coil actuator.

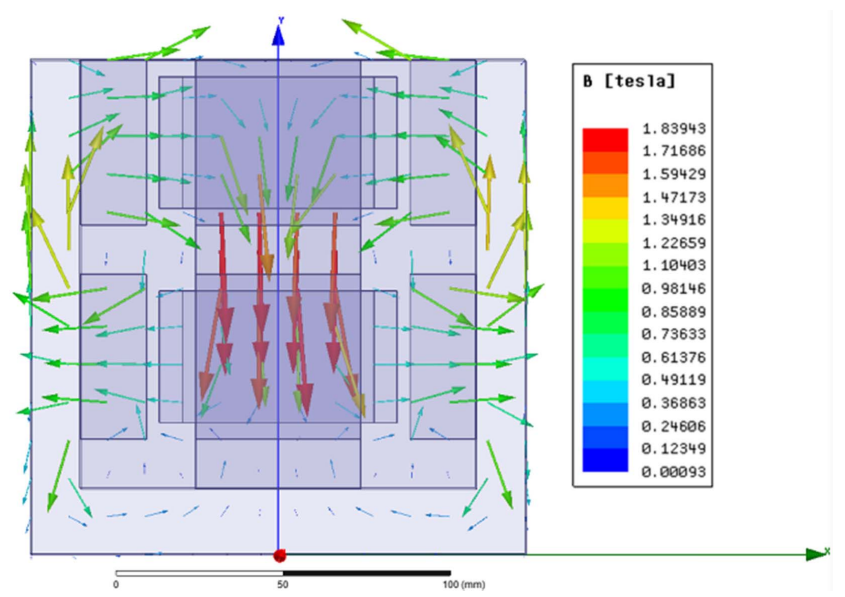

Fig. 2. (Color online) Magnetic field in the cross-section of the VCA.

be axis-symmetric, it can be configured as a circular-type or a rectangular-type. Though a circular type incurs no coil loss since the entire coil is within the magnetic field, it is difficult to construct a radially-magnetized magnet. Alternatively, several segmented radial magnets can be used, but this increases the manufacturing cost. Though coil loss is incurred at the four corners in case of rectangular type, it is cost-effective and easy to assemble. A rectangular-type VCA has two rectangular coils and eight magnets. Figure 2 shows the magnetic field at the cross-section of the proposed VCA having two closed paths of magnetic flux. Horizontal magnetic flux is produced in the air-gap between the permanent magnets and center yoke. The coils placed in this air gap (as shown in Fig. 1) moves up and down by the Lorentz's law. The upper and lower coils have opposite winding 


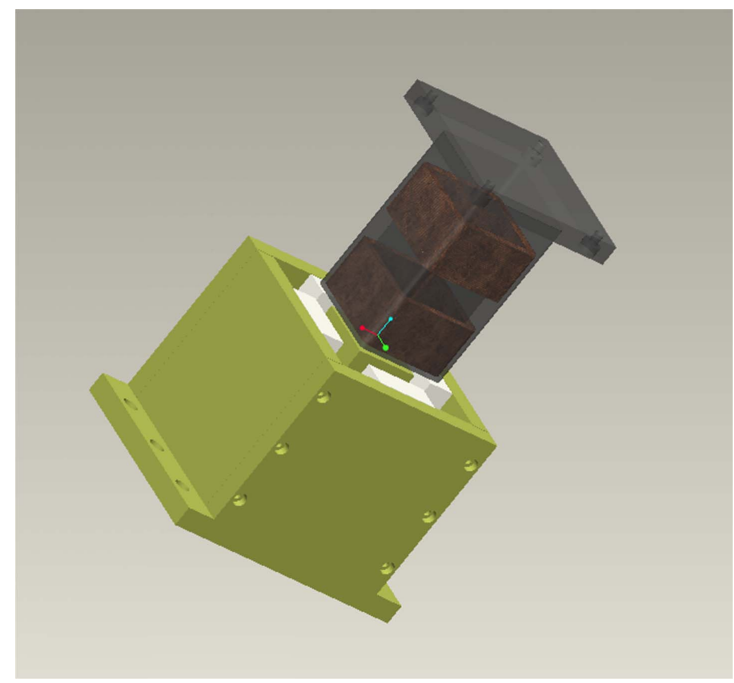

Fig. 3. (Color online) Coil block and magnet assembly (exploded view).

directions so as to generate force in the same direction. Figure 3 shows the proposed 3D concept of the rectangular-type VCA. The two coils are molded together using molding epoxy. The permanent magnets are bonded to side yokes using adhesives. All permanent magnets are

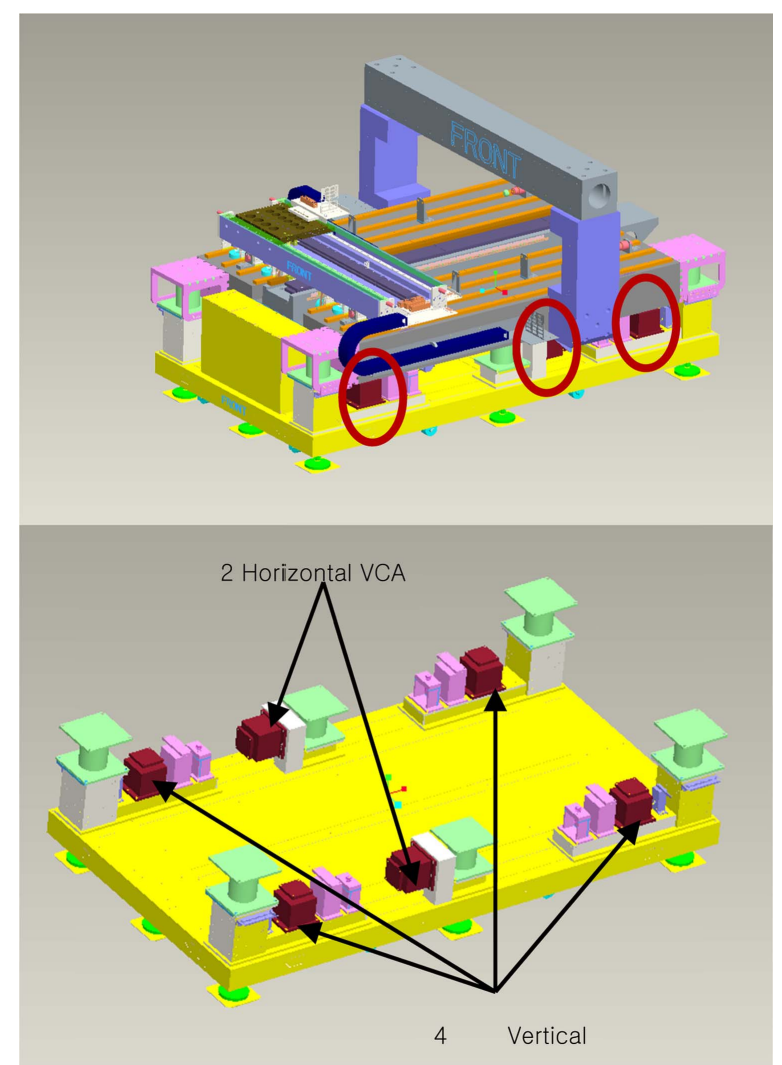

Fig. 4. (Color online) Layout of a 5-DOF active isolated system. identical.

In order to control frame vibration of the stage, two VCAs are installed horizontally at the middle of the frame, and four VCAs are installed vertically at four corners of the frame as shown in Fig. 4. The stage's footprint is about $2000 \mathrm{~mm} \times 3000 \mathrm{~mm} \times 1000 \mathrm{~mm}$. Because of this multi-degrees-of-freedom configuration, the VCA must have enough clearance between the coil assembly and the magnet assembly to prevent any collision, although a large clearance reduces the magnetic flux through the coil and the subsequently generated force. The proposed VCA was designed to have a clearance of $\pm 4 \mathrm{~mm}$ considering the allowable motion of the passive isolators.

\section{Modeling and Optimization}

This section describes the geometric and electromagnetic model of the proposed VCA and discusses the optimization of its design.

\subsection{Geometric model and material}

Figure 5 shows the geometric model of a quarter part of the proposed VCA. The parameters presented in Fig. 5 were used to describe a magnetic circuit model. In the interest of simplicity, we assume that the width of coil block is proportional to the length of magnet, $l_{m}$, and that the distance between the two magnets is same as the distance between the lower magnet (the right magnet in Fig. 5) and the bottom yoke as $t_{b}$. Also, the length and width of the magnet are same as $l_{m}$.

For magnets assembly, we have used rare earth permanent magnets (N-50M grade NdFeB, STARGROUPIND). The permanent magnet has a remanence of $1.45 \mathrm{~T}$, and a coercive force greater than $912 \mathrm{kA} / \mathrm{m}$. The yokes are made of carbon steel (JIS S10C) with yoke saturation assumed as $1.8 \mathrm{~T}$.

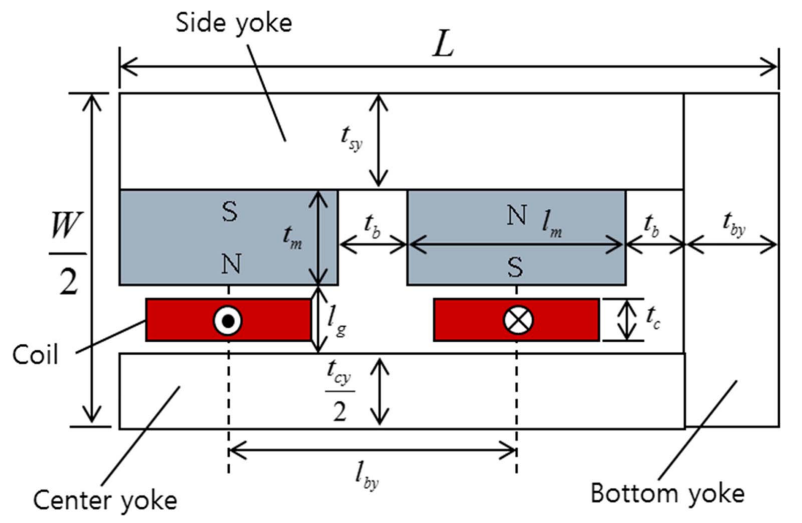

Fig. 5. (Color online) Geometric model for a quarter part of the proposed VCA. 


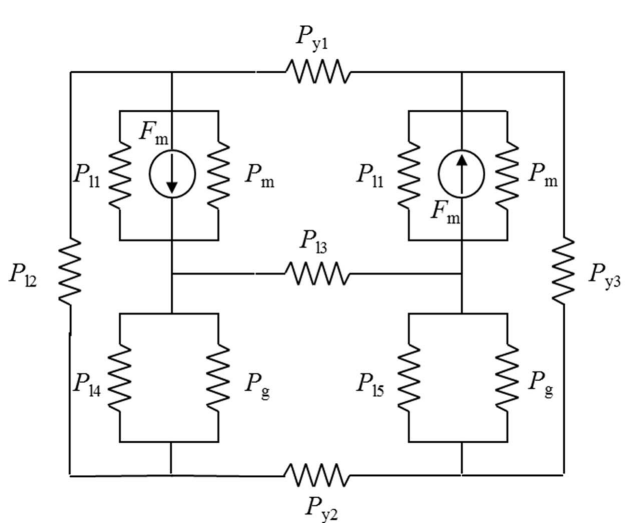

(a)

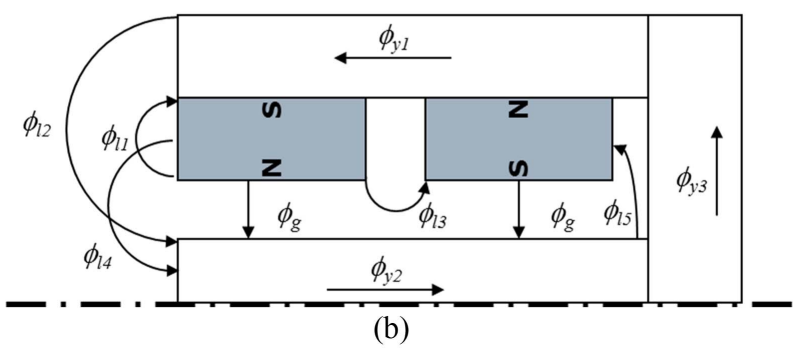

Fig. 6. (Color online) Magnetic model (a) magnetic circuit model (b) leakage flux.

\subsection{Magnetic circuit model}

An electromagnetic model is required to calculate the force generated by the VCA. Though the finite element method (FEM) is accurate, it is computationally too intensive to perform iterative calculations for optimization. The permeance method $[13,14]$ models a magnetic circuit in a way similar to that used for an electrical circuit. Permeance is the reciprocal of reluctance in magnetic circuit and is determined by the shape of the part and the permeability of material. Figure 6(a) depicts a magnetic circuit model of the quarter part of the proposed VCA using the permeance method. In Fig. 6(a), $P_{\mathrm{y} 1}, P_{\mathrm{y} 2}$, and $P_{\mathrm{y} 3}$ are the permeances of the side yoke, the center yoke and the bottom yoke, respectively. $P_{11}, P_{12}, P_{13}, P_{13}, P_{14}$, and $P_{15}$ are the leakage permeances. $P_{\mathrm{m}}$ and $F_{\mathrm{m}}$ are the permeance and the magnetomotive force of the permanent magnet, respectively. $P_{\mathrm{g}}$ is the permeance of the air gap. The leakage flux, $\phi_{\mathrm{li}}$ corresponding the leakage permeance, $P_{\mathrm{li}}$ is shown in Fig. 6(b). Table 1 describes the equations of the leakage permeance. It is very important to model proper leakage permeances because they greatly affects the model accuracy. Using the Kirchhoff's law, we can calculate the magnetic flux, $\phi_{g}$, at the air gap. The magnetic flux density in the air gap is given as

$$
B_{g}=\frac{\phi_{g}}{l_{m}^{2}+1.22 \frac{\pi}{2} l_{m} l_{g}}
$$

where $l_{m}$ is the length of the magnet, and $l_{g}$ is the gap between the magnet and the center yoke. The electromagnetic force of the VCA can then be calculated by the Lorentz's law using this magnetic flux density, coil turns and current.

\subsection{Design optimization}

Performance specifications such as generated force, power consumption, and size of the VCA are determined by the geometry of magnet and coil. Among the geometric parameters shown in Fig. 5, we have chosen four design parameters that significantly affect these performance specifications. The first chosen parameter is the length of the permanent magnets, $l_{m}$, as it is related to the area of the magnetic field in which the coil resides. Since the saturation of the magnetic flux through the yoke reduces the magnetic flux in the air gap, the thickness of the center yoke, $t_{c y}$, was chosen as the second design parameter. Next, we choose the thickness of coil block, $t_{c}$, and the wire diameter, $d_{c}$, because of the dependence of the number of coil turns and the coil resistance on these parameters. In addition to the geometric design parameters,

Table 1. Leakage permeances.

\begin{tabular}{cll}
\hline \hline Permeance & \multicolumn{1}{c}{ Equation } & Description \\
\hline$P_{11}$ & $P_{11}=4 \mu_{0} \frac{l_{m}}{\pi}$ & Leakage of the permanent magnet itself \\
$P_{12}$ & $P_{12}=0.26 \cdot \mu_{0} \cdot l_{m}$ & Leakage between the side yoke and the center yoke \\
$P_{13}$ & $P_{13}=0.26 \cdot \mu_{0} \cdot l_{m}+2 \cdot 0.26 \cdot \mu_{0} \cdot t_{m}$ & Leakage between two permanent magnets \\
$P_{14}$ & $P_{14}=3 \cdot \mu_{0} \cdot \frac{l_{m}}{\pi} \log \left(1+\frac{t_{m}}{l_{g}}\right)+3 \cdot 0.26 \cdot \mu_{0} \cdot l_{m}$ & Leakage between the left magnet and the center yoke (from Fig. 6(b)) \\
$P_{15}$ & $P_{15}=2 \cdot \mu_{0} \cdot \frac{l_{m}}{\pi} \log \left(1+\frac{t_{m}}{l_{g}}\right)+2 \cdot 0.26 \cdot \mu_{0} \cdot l_{m}$ & Leakage between the right magnet and the center yoke (from Fig. 6(b))
\end{tabular}


Table 2. Constraints for optimization.

\begin{tabular}{llcc}
\hline \hline No. & Constraint parameter & Min & Max \\
\hline C1 & Force $(\mathrm{N})$ & 573 & - \\
C2 & Total width $(\mathrm{mm})$ & - & 150 \\
C3 & Yoke saturation $(\mathrm{T})$ & - & 1.8 \\
C4 & Driving voltage & - & 80 \\
C5 & Coil diameter, $d_{c}(\mathrm{~mm})$ & 0.3 & 1 \\
C6 & Coil thickness, $t_{c}(\mathrm{~mm})$ & 3 & 15 \\
C7 & Yoke thickness & 30 & 50 \\
C8 & Current $(\mathrm{A})$ & 2 & 10 \\
C9 & Magnet length, $l_{m}(\mathrm{~mm})$ & 30 & 70 \\
\hline
\end{tabular}

we add the last design parameter: the maximum current, $I$. The maximum current is directly related to the maximum force and power consumption of the VCA.

The power consumption of the VCA should be as low as possible in order to reduce heat generation from the coils and the capacity requirement of a current amplifier. In this paper, we have tried to minimize the power consumption with the constraints of force requirement, size limitation, yoke saturation, and driving voltage limitation using the design optimization process [15]. The optimization process is implemented by a sequential quadratic
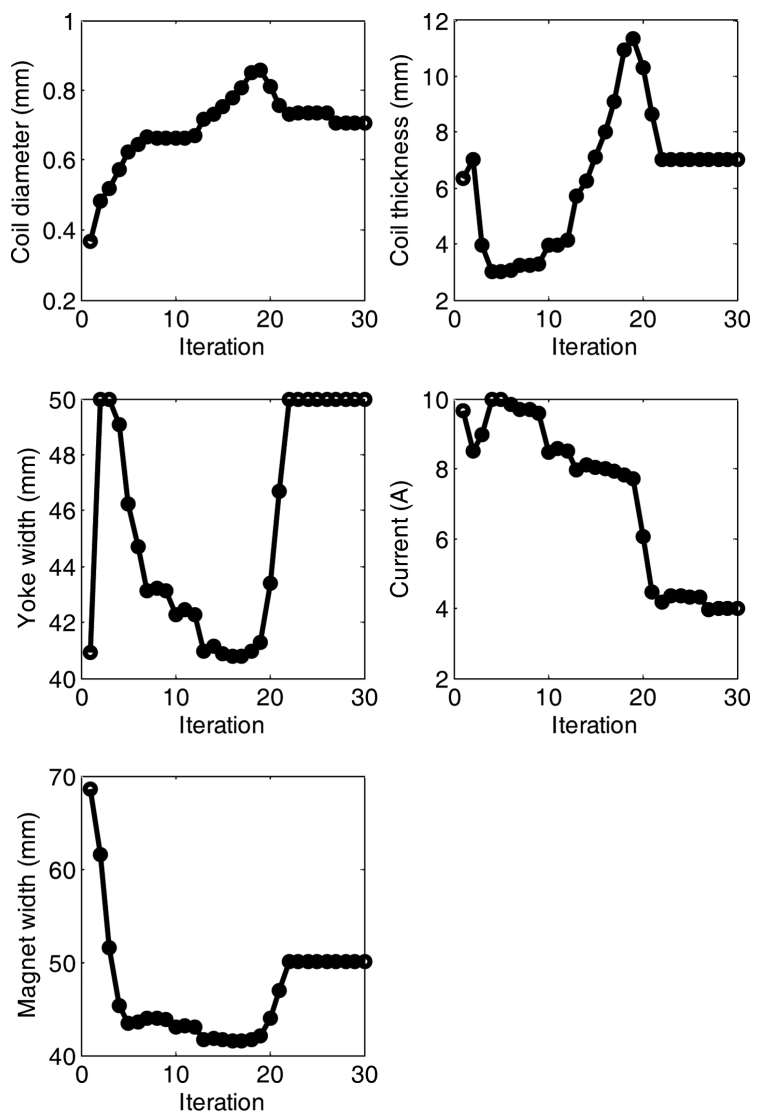

Fig. 7. Convergence of design parameters.

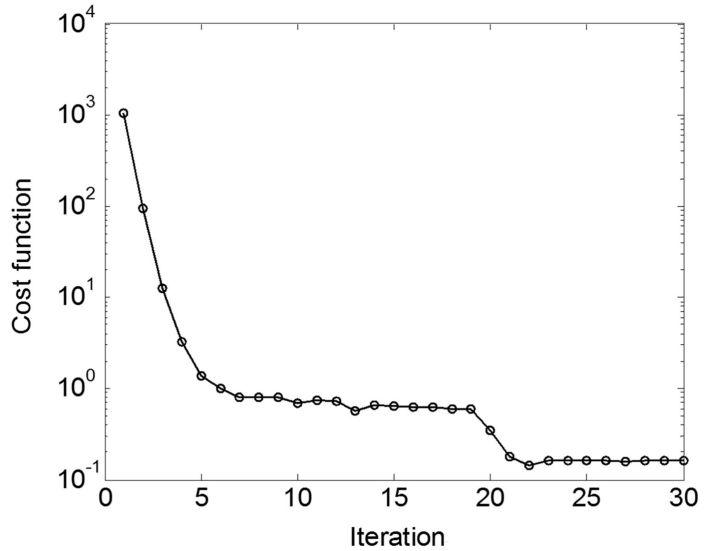

Fig. 8. Convergence of the cost function.

programming (SQP) algorithm. The cost function of optimization was set to the square of the reciprocal of power consumption. The power consumption is given as

$$
P_{V C A}=R_{c} I^{2}+F_{r e q} v
$$

where $R_{c}$ is the resistance of the coil, $F_{\text {req }}$ is the maximum force of the VCA, and $v$ is the operating speed that induces a back electromotive force (EMF) voltage. The constraints are listed in Table 2.

The converged design parameters and cost function according to optimization iterations are shown in Fig. 7 and Fig. 8. The design parameters from the optimization are summarized in Table 3. The total size of the VCA was $150 \mathrm{~mm}(\mathrm{~W}) \times 150 \mathrm{~mm}(\mathrm{D}) \times 160 \mathrm{~mm}(\mathrm{H})$. For reference, we have performed the finite element model (FEM) analysis (Maxwell, ANSYS) for calculation of the force constant with these optimized parameters. The FEM analysis was found to produce a force constant of 138.2 N/A, which is well-matched with the magnetic circuit model. However, since not much variation is available in the diameters of the commercially-available copper wires, we select the nearest possible value, $0.66 \mathrm{~mm}$. The final results using the modified coil diameter were re-calculated using FEM analysis and are shown in Table 3. Because of the smaller coil diameter, a larger force constant was obtained, but the power consumptions were almost similar to that of the optimized results. The FEM analysis gave a force constant of 163.9 N/A with a power of $322 \mathrm{~W}$.

Next, we analyzed the aspect of heat generation from the coil using the thermal FEM analysis on the temperature distribution. Generally, the enamel coating of the coil can withstand below $100{ }^{\circ} \mathrm{C}$ and the $\mathrm{N}-50 \mathrm{M}$ magnet can work up to $80{ }^{\circ} \mathrm{C}$. When continuous current of $1.15 \mathrm{~A}$ ( $33 \%$ duty of a maximum current of $3.5 \mathrm{~A}$ ) flows, the temperature of the hottest spot was found to be about $71{ }^{\circ} \mathrm{C}$ (as shown in Fig. 9). In this FEM model, we assume that all 
Table 3. Optimization results.

\begin{tabular}{|c|c|c|c|}
\hline & & Optimized results & Final decision (FEM) \\
\hline \multirow{5}{*}{ Design parameters } & Coil diameter $(\mathrm{mm})$ & 0.71 & 0.66 \\
\hline & Coil thickness (mm) & 7.0 & 7.0 \\
\hline & Center yoke thickness (mm) & 50.0 & 50.0 \\
\hline & Maximum current (A) & 4.0 & 3.5 \\
\hline & Magnet width (mm) & 50.1 & 50.0 \\
\hline Cost function & Consumed power $(\mathrm{W})$ & 320 & 322 \\
\hline Ref. performance index & Force constant (N/A) & 143.1 & 163.9 \\
\hline
\end{tabular}

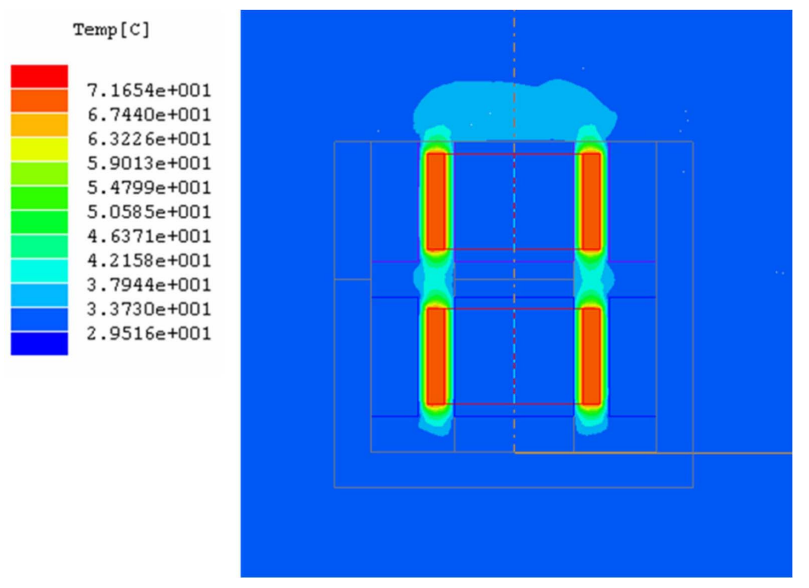

Fig. 9. (Color online) Temperature distribution of the VCA.

heat is dissipated by natural convection with a transfer coefficient $h=10 \mathrm{~W} /\left(\mathrm{m}^{2} \cdot{ }^{\circ} \mathrm{C}\right)$. This assumption represents a condition much worse than the actual conditions. In a real situation, the operating duty is expected to be much smaller than $33 \%$ and the operation is safe even if the heat convection varies according to structure and temperature.

As discussed earlier, the gap between the coil block and the magnet assembly cannot be constantly maintained due to radial vibration by the frame. Using FEM analysis, we evaluate the $z$ - and $x$-directional forces (as shown in Fig. 10) when the coil block is deviated horizontally from the nominal position. Over a quarter of working space $([x, y]$ $=[0 \mathrm{~mm}, 4 \mathrm{~mm}])$, the $z$-directional force is nearly equal to the nominal value as shown in Fig. 10(a). The $x$ directional force, which is a parasitic force, is kept less than about $13 \%$ of the $z$-directional force against the variations of the coil location as shown in Fig. 10(b).

\section{Experimental Results}

This section discusses the experimental results obtained with the proposed VCA. Electric properties and electro-

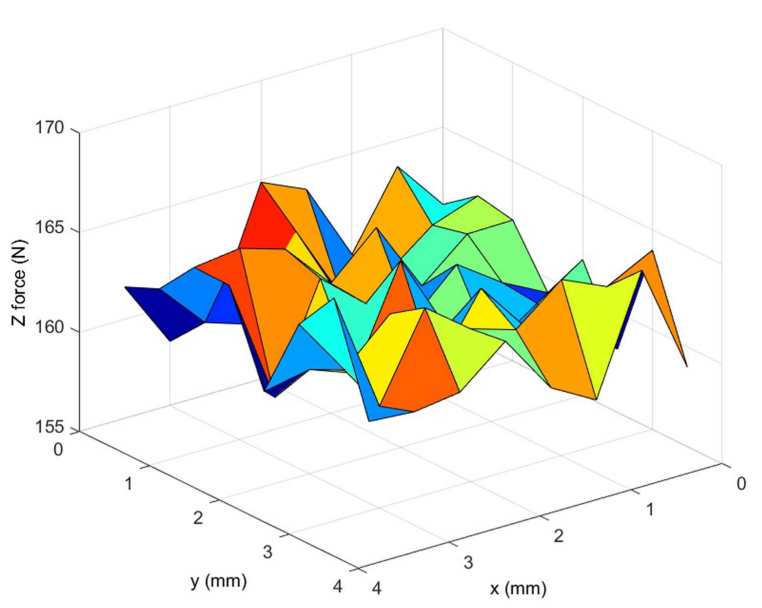

(a)

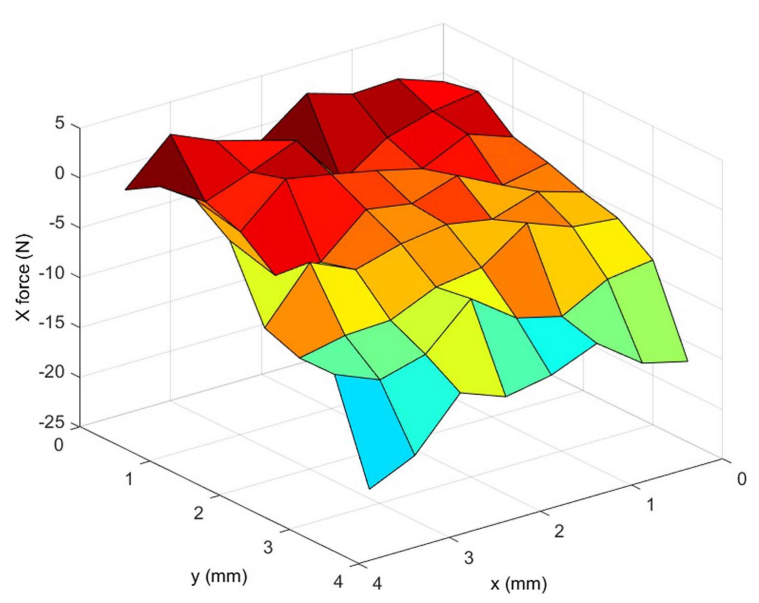

(b)

Fig. 10. (Color online) Force variations (a) axial force (z), (b) parasitic force $(\mathrm{x})$.

magnetic force were measured.

\subsection{Resistance and inductance}

The measured resistance of the coil at $25^{\circ} \mathrm{C}$ was about $25 \Omega$. This value slightly deviates from the theoretically- 


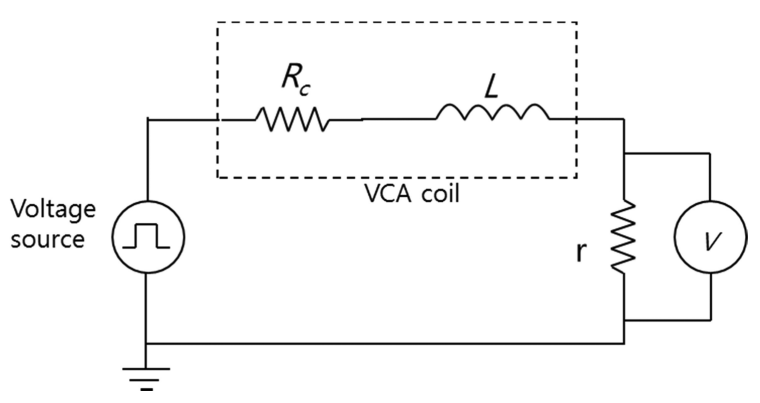

Fig. 11. Test circuit for measurement of coil inductance.

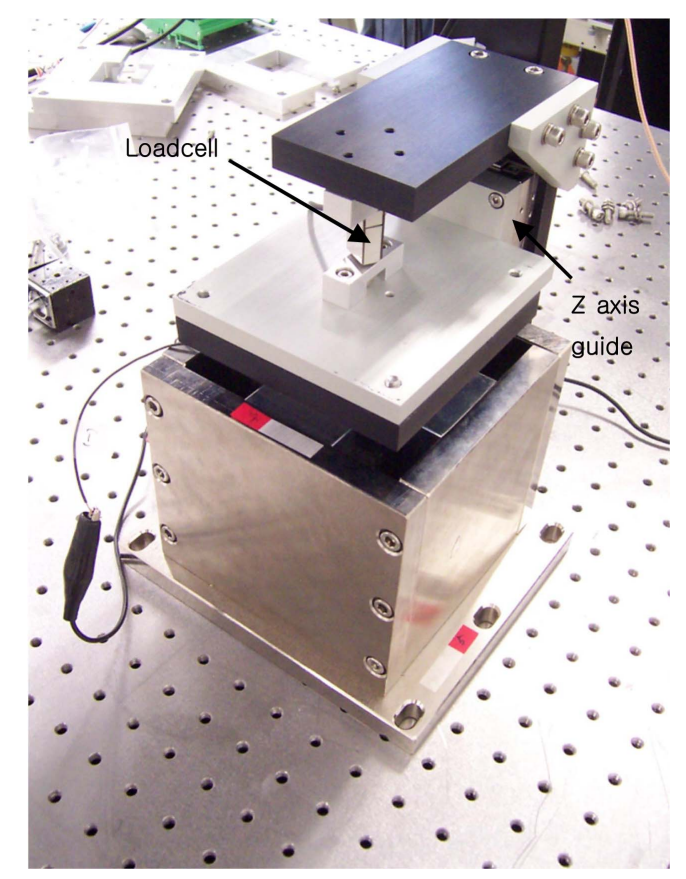

Fig. 12. (Color online) Experimental setup for force test.

estimated value of the resistance $\left(R_{c}\right)$ of $23 \Omega$. The change in the coil diameter during the winding process may be the reason behind this deviation.

Next, the inductance of the coil was measured experimentally. The inductance is usually quite small and is often ignored. However, since the proposed mighty VCA has many coil turns, the inductance is expected to be large. We also studied whether the electrical time constant would affect the bandwidth of the VCA. As shown in Fig. 11 , we connected the current source to the coil with the additional resistance, $r$, of $684 \Omega$. The electrical time constant, given by $L /\left(R_{c}+r\right)$, was then measured with the rectangular-wave current source, and was found to be 174

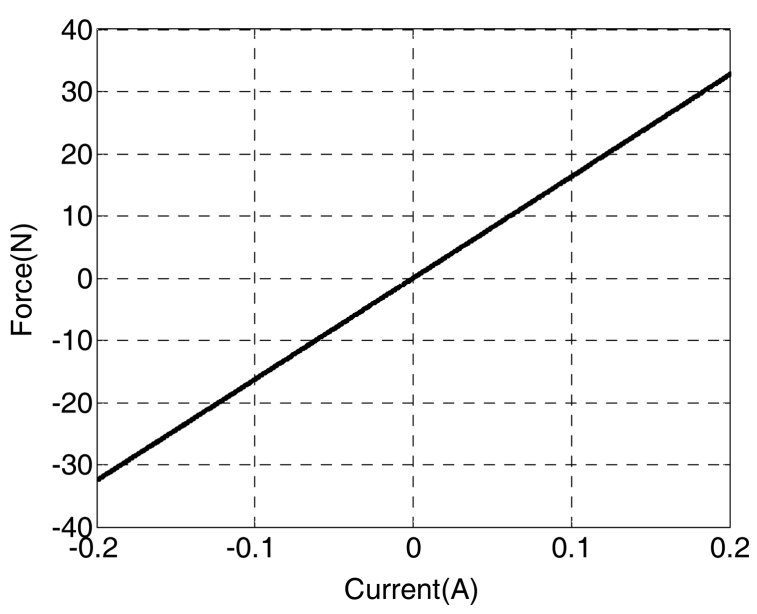

Fig. 13. Measured force with respect to the input current.

$\mu$ s. Thus, the estimated inductance of the coil was found to be $123 \mathrm{mH}$. When the VCA operates alone, the electrical time constant is $4.9 \mathrm{~ms}$. Since the control bandwidth of the active isolated frame for the proposed VCA is around $20-30 \mathrm{~Hz}$, the large inductance of $123 \mathrm{mH}$ may not be a problem.

\subsection{Force evaluation}

The manufactured VCA and the test setup are shown in Fig. 12. The coil block is guided in the vertical direction by a sliding stage to set the coil at the nominal position. A load cell (CSBA-10LS, CAS) measures the pushing force of the coil assembly. Table 4 shows the measured force constants from eight VCAs. The average force constant is $166.8 \mathrm{~N} / \mathrm{A}$. As the final design result was about $164 \mathrm{~N} / \mathrm{A}$, the manufacturing error is found to be less than $1.5 \%$. Further, it is noted that the VCAs are fabricated with an excellent uniformity with a standard deviation error of $0.9 \%$. Figure 13 depicts the measured current-to-force relationship for one VCA and displays perfect bidirectional linearity.

\section{Conclusions}

This paper discusses the development of a mighty voice coil actuator for the reaction compensation of the stage. To allow radial motion, the proposed VCA was designed to have a sufficient clearance of $4 \mathrm{~mm}$. Further, the VCA was design as an axis-symmetric rectangular structure for simple and economic fabrication. Through optimized design

Table 4. Measured force constant for all manufactured VCA.

\begin{tabular}{ccccccccccc}
\hline \hline VCA Number & 1 & 2 & 3 & 4 & 5 & 6 & 7 & 8 & Average & Standard deviation \\
\hline Force constant (N/A) & 163 & 167 & 168 & 167 & 167 & 167 & 168 & 167 & 166.8 & $1.48(0.9 \%)$ \\
\hline
\end{tabular}


and FEM analysis, the proposed VCA was found to produce a maximum force of $574 \mathrm{~N}$ and a corresponding power consumption of $322 \mathrm{~W}$. Experimental results showed that the fabricated VCA has good bi-directional linearity and uniform fabrication quality. The active compensation of frame vibration plays a key role in the next generation of large-scale flat-panel manufacturing. By reducing the residual vibration, the stage can be operated with a smaller dwell time even at a faster speed, which in turn, improves the overall productivity.

\section{Acknowledgment}

This work was supported, in part, by the Ajou University and the National Research Foundation of Korea (NRF) Grant No. 2014R1A2A1A10052344 funded by the Korean government (MSIP).

\section{References}

[1] A. M. Rankers, Ph.D. dissertation, Univ. Twente, Enschede, The Netherlands, 67 (1997).

[2] CANON, US patents, US20050217956 (2005).

[3] Nikon, US patents, US20050248744 (2005).
[4] Hirokazu Yoshioka and Nobuyoshi Murai, Proceedings of the 7th International Workshop on Accelerator Alignment, SPring-8 (2002).

[5] Yi-De Chen, Chyun-Chau Fuh, and Pi-Cheng Tung, IEEE Trans. Magn. 41, 1149 (2005).

[6] Rahul Banik and Dae-gab Gweon, Sensors and Actuators A 137, 236 (2007).

[7] Y. Nakamura, M. Nakayama, M. Yasuda and T. Fujita, Smart Materials and Structures, 15, 1133 (2006).

[8] Shinji Wakui, Precision Engineering, 27, 170 (2003).

[9] K. Mori, T. Munemoto, H. Otsuki, Y. Yamaguchi, and K. Akagi, IEEE Trans. Magn. 27, 5298 (1991).

[10] Chien-Sheng Liu, Psang-Dain Lin, Po-Heng Lin, ShunSheng Ke, Yu-Hsiu Chang, and Ji-Bin Horng, IEEE Trans. Magn. 45, 155 (2009).

[11] Young-Man Choi and Dae-Gab Gweon, IEEE/ASME Trans. Mech. 16, 925 (2011).

[12] Q. Y. Wu, Qiang Wang, Qi Peng, Ge Ren, and C. Y. Fu, Opto-Electronic Engineering 31, 15 (2004).

[13] D. C. Hanselman, McGraw-Hill, Inc. (1994).

[14] Liu, Yu, Ming Zhang, Yu Zhu, Jin Yang, and Badong Chen, IEEE Trans. Magn. 47, 2247 (2011).

[15] M. G. Lee and D.-G. Gweon, J. Magn. Magn. Mater. 218, 336 (2004). 